\title{
Thyroid Hormone Secretion Process
}

National Cancer Institute

\section{Source}

National Cancer Institute. Thyroid Hormone Secretion Process. NCI Thesaurus. Code C41495.

Thyroid Hormone Secretion Induction consists of initiation of activities involved in release into the bloodstream by thyroid gland follicular cells of closely related iodine-containing triiodothyronine and thyroxine (thyroid hormones) formed from thyroglobulin that increase the rate of cell metabolism and are essential for central nervous system maturation. Alteration of positive or negative feedback mechanisms can affect hormone release. 